\title{
Laboratório de Informática, Aprendizagem e Gestão (LIAG)
}

\author{
Marcos Augusto Francisco Borges ${ }^{1}$ \\ ${ }^{1}$ Faculdade de Tecnologia - Universidade Estadual de Campinas (UNICAMP) \\ R. Paschoal Marmo, 1888 - CEP:13484-332 - Jd. Nova Itália - Limeira, SP \\ marcosborgeseft.unicamp.br
}

\section{Objetivo(s) do Grupo}

Missão: Integrar o estudo das áreas de informática, aprendizagem e gestão de modo a buscar resultados superiores e diferenciados nas três áreas.

Visão: Ser reconhecido como um laboratório com resultados acadêmicos relevantes e com forte interação com a comunidade.

2. Universidade participante

Faculdade de Tecnologia - FT; Universidade Estadual de Campinas - UNICAMP

3. Pesquisadores/Desenvolvedores envolvidos

Coordenador: Marcos Augusto Francisco Borges

Mestrandos: Erika Yamagishi; Marco Cantanhede; Ana L. S. de Souza

Iniciação Científica: Elenilson dos Santos Pereira; Lucas Leonardo Padula; Romulo Messias Silva Souza; Matheus Teles de Freitas

Bolsistas: Jennifer de Oliveira Marcelo; Antonio Augusto Barbosa Baptista; Amanda Oliveira Santos; Karoline Neves Rocha;Tiago Filipe Dan Borges de Oliveira Colaboradores voluntários: Roberta Matsunaga

Parceiros:

Profa. Dra. Gisela de Aragão Umbuzeiro, Laboratório de Ecotoxicologia e Microbiologia Ambiental "Dr Abílio Lopes“, FT-UNICAMP;

Marcia Aparecida Piccoloto Matta, Margareth Ozelo, IHTC Unidade de Hemofilia Cláudio Luis Pizzigatti Corrêa, INCT do Sangue Hemocentro UNICAMP;

Núcleo de Informática Aplicada à Educação - NIED - UNICAMP

4. Principais projetos (concluídos/em andamento) e fomentos recebidos

Coordenação do CBIE 2013;

Improve MSK status for PWH in Brazil by Measuring, Analysing, Disseminating and Educating: Novo Nordisk Haemophilia Foundation - 2011 a 2014;

Desenvolvimento do jogo DaphniaWorld: bolsistas de iniciação científica, 2011 até hoje;

Sistema de Apoio ao Aprendizado de Genética: FAPESP - 2009 a 2011 (projeto continua até hoje com bolsistas de iniciação científica);

Construção de uma GoGo Board e avaliação de seu uso para a aprendizagem: FAEPEXUNICAMP - 2008 a 2009;

Criação e condução da LIGA Robocode - vários patrocinadores - 2011 até hoje 